\title{
Linearized Kompaneetz equation as a relativistic diffusion
}

\author{
Z. Haba \\ Institute of Theoretical Physics, University of Wroclaw, \\ 50-204 Wroclaw, Plac Maxa Borna 9, Poland, \\ email:zhab@ift.uni.wroc.pl \\ PACS numbers:05.10.Gg,05.20.Dd,98.80.Jk
}

November 3, 2018

\begin{abstract}
We show that Kompaneetz equation describing photon diffusion in an environment of an electron gas, when linearized around its equilibrium distribution, coincides with the relativistic diffusion discussed in recent publications. The model of the relativistic diffusion is related to soluble models of imaginary time quantum mechanics. We suggest some non-linear generalizations of the relativistic diffusion equation and their astrophysical applications (in particular to the Sunyaev-Zeldovich effect).
\end{abstract}

\section{Introduction}

An evolution in time of a stream of particles can in general be described by the Boltzmann equation. In this paper we are interested in an evolution of a relativistic gas of massless particles . The relativistic Boltzmann equation is the standard tool in a study of the evolution of the photon in a gas of charged particles [1][2]. A diffusion approximation to the non-relativistic Boltzmann equation has been applied in many models of the transport phenomena [3]. The diffusion approximation to the relativistic Boltzmann equation is applied in the heavy ion collisions and the theory of quark-gluon plasma [4][5]. A long time ago Kompaneetz [6] ( see also [7]) derived a non-linear diffusion equation applicable to the photon propagation in an environment of an electron gas. The equation describes an evolution of a beam of photons disturbed by Compton scattering and Bremsstrahlung. These are the processes widely studied in astrophysics [1][8][9].

There seems to be the unique way to approximate the non-relativistic transport phenomena by the diffusion. The concept of a relativistic diffusion did 
not achieve such a generally accepted consensus. Various versions of the relativistic diffusion are discussed in [10][11][12][13][14][15][16][17](for a review and further references see [18][19]). In refs.[10][11] the relativistic Brownian motion is uniquely defined by the requirement that this is the diffusion whose four momentum stays on the mass-shell and the generator of the diffusion is the second order relativistic invariant differential operator. Starting from this basic assumption we discussed in [20] friction terms leading to an equilibrium. In [21] we studied a diffusion of elementary particles with spin.

In this paper we show (sec.3) that if the Kompaneetz equation is expanded around its equilibrium ( Bose-Einstein) solution and non-linear correction terms are neglected then the resulting diffusion equation is the same as the relativistic diffusion with friction. First, in sec.2 we formulate the relativistic diffusion equation for massless particles in a form appropriate for a comparison with the Kompaneetz equation. In sec. 4 we show that the linearized Kompaneetz equation can be solved applying methods of quantum mechanics because the relativistic diffusion can equivalently be described as quantum mechanical evolution in an imaginary time. Finally, in sec.5 we suggest a generalization of the Kompaneetz equation to a moving frame and a background gravitational field. This is a non-linear equation taking into account the quantum statistics of bosons whose linear approximation coincides with the (linear) relativistic diffusion discussed in [17](without friction) and in [22] (with friction).

\section{The relativistic diffusion of massless particles}

Following Schay[10] and Dudley[11] we define the generator $\triangle_{m}$ of the relativistic diffusion as the $O(3,1)$ invariant second order differential operator on the mass-shell $\mathcal{H}_{m}$

$$
p^{2}=p_{0}^{2}-p_{1}^{2}-p_{2}^{2}-p_{3}^{2}=m^{2} c^{2} .
$$

The limit $m \rightarrow 0$ of $\triangle_{m}$ is divergent. In order to obtain a finite result we take the limit $m \rightarrow 0$ of $m^{2} \triangle_{m}$. This limit coincides with another definition of the diffusion [21] as the process generated by $M_{\mu \nu} M^{\mu \nu}$ where $M_{\mu \nu}$ are generators of the Lorentz group (the formula for a diffusion of a massless particle has been also derived in [23] as a continuum limit of discrete dynamics of space-time, see also [24]).

Choosing $\mathbf{p}$ (boldface type letters denote three dimensional vectors) as coordinates on $\mathcal{H}_{0}$ we obtain as the limit $m \rightarrow 0$ of $m^{2} \triangle_{m}$ the operator

$$
\triangle_{H}=p_{j} p_{k} \partial^{j} \partial^{k}+3 p_{k} \partial^{k}
$$

here $k=1,2,3$ and $\partial^{j}=\frac{\partial}{\partial p_{j}}$. The relativistic diffusion equation in the momentum space (a relativistic analog of the Brownian motion) reads

$$
\partial_{\tau} \phi_{\tau}=\frac{1}{2} \gamma^{2} \triangle_{H} \phi_{\tau}
$$


$\gamma$ is a diffusion constant. The photon as a massless particle is a typically relativistic object. At the same time this is a quantum system with an internal angular momentum (spin). In this paper we neglect the spin. In [21] we discuss the diffusion of particles with a spin (the helicity in the massless case). It is shown in [21] that if $m=0$ then the dissipative part of the evolution does not depend on the helicity . Hence, the neglect of spin in this paper is justified.

We add a drag term

$$
X=Y_{j} \frac{\partial}{\partial p_{j}}+\gamma^{2} R_{j} \frac{\partial}{\partial p_{j}}+p_{\mu} \frac{\partial}{\partial x_{\mu}}
$$

to the diffusion (3). The vector field $R$ defines a perturbation (friction) of the relativistic dynamical system (at $\gamma=0$ )

$$
\begin{aligned}
& \frac{d p_{j}}{d \tau}=Y_{j}, \\
& \frac{d x^{\mu}}{d \tau}=p^{\mu}
\end{aligned}
$$

where $\mu=0,1,2,3$ and from eq.(1) (for $m=0$ ) we have $p^{0}=|\mathbf{p}| \cdot \tau$ is an affine time parameter describing relativistic invariant dynamics. Its relation to the laboratory time $x^{0}$ follows from the zeroth component of eq.(5). We set $Y=0$ till sec.5 where the vector $Y$ will describe a geodesic motion on a manifold.

Let

$$
\mathcal{G}=\frac{1}{2} \gamma^{2} \triangle_{H}+X
$$

Now, the diffusion equation reads

$$
\partial_{\tau} \phi_{\tau}=\mathcal{G} \phi_{\tau}
$$

We are interested in the behavior of the relativistic dynamics at large values of $\tau$. In particular, whether the diffusion generated by $\mathcal{G}$ can have an equilibrium limit. We say that the probability distribution $d x d \mathbf{p} \Phi$ is the invariant measure for the diffusion process if the statistical mean values are $\tau$-independent (there may remain the dependence on $x^{0}$ )

$$
\int d x d \mathbf{p} \Phi(x, \mathbf{p}) \phi_{\tau}(\mathbf{p}, x) \equiv \int d x d \mathbf{p} \Phi_{\tau}(\mathbf{p}, x) \phi(\mathbf{p}, x)=\text { const } .
$$

Here, the evolution of $\Phi$ is defined by

$$
\partial_{\tau} \Phi_{\tau}=\mathcal{G}^{*} \Phi_{\tau}
$$

and $\mathcal{G}^{*}$ is the adjoint of $\mathcal{G}$ in $L^{2}(d \mathbf{p} d x)$ (the Hilbert space of square integrable functions; the Lebesgue measure $d x d \mathbf{p}$ is not Lorentz invariant, the factor $|\mathbf{p}|^{-1}$ necessary for the Lorentz invariance is contained in $\Phi$, see [20][22]). 
The $\tau$-invariance (8) is equivalent to

$$
\mathcal{G}^{*} \Phi=0 .
$$

Eq.(10) defines a transport equation in the laboratory time $x^{0}$.

We denote by $\Phi_{E R}$ the $x$-independent solution of eq.(10). Then, $R$ can be expressed in terms of $\Phi_{E R}$

$$
R_{j}=\frac{1}{2} p_{j}+\frac{1}{2} p_{j} p_{k} \partial^{k} \ln \Phi_{E R} .
$$

We assume that $\Phi_{E R}$ is a function of the energy $p_{0} c$ multiplied by a constant $\beta$ of the dimension inverse to the dimension of the energy $\left(\beta=\frac{1}{k T}\right.$ in the conventional notation, where $T$ denotes the temperature). In such a case from eq.(11)

$$
R_{j}=\frac{1}{2} p_{j}+\frac{1}{2} \beta c p_{j}|\mathbf{p}|\left(\ln \Phi_{E R}\right)^{\prime}(c \beta|\mathbf{p}|) .
$$

Now, eq.(7) reads

$$
\partial_{\tau} \phi_{\tau}=\frac{1}{2} \gamma^{2} \triangle_{H} \phi_{\tau}+\frac{1}{2} \gamma^{2} p_{j}\left(1+\beta c|\mathbf{p}|\left(\ln \Phi_{E R}\right)^{\prime}\right) \partial^{j} \phi_{\tau} .
$$

Let us consider an initial probability distribution of the form $\Phi=\Phi_{E R} \Psi$. Then, from eq.(9) we obtain its evolution

$$
\Phi_{\tau}=\Phi_{E R} \Psi_{\tau}
$$

as a solution of the equation

$$
\partial_{\tau} \Psi_{\tau}=\frac{1}{2} \gamma^{2} p_{j} p_{k} \partial^{j} \partial^{k} \Psi_{\tau}+2 \gamma^{2} p_{j} \partial^{j} \Psi_{\tau}+\frac{1}{2} \gamma^{2} p_{j} p_{k}\left(\partial^{j} \ln \Phi_{E R}\right) \partial^{k} \Psi_{\tau}-p_{\mu} \partial_{x}^{\mu} \Psi_{\tau}
$$

(derivatives over the space-time coordinates have an index $x$, till sec.5 we restrict ourselves to the diffusion in momentum space, hence we drop the spatial derivatives). The diffusion generator (2) is degenerate. This is easy to see if we express it in the spherical coordinates on $\mathcal{H}_{0}$

$$
p_{0}=r
$$

$p_{1}=r \cos \phi \sin \theta, p_{2}=r \sin \phi \sin \theta, p_{3}=r \cos \theta$. In these coordinates

$$
\triangle_{H}=r^{2} \frac{\partial^{2}}{\partial r^{2}}+3 r \frac{\partial}{\partial r}=\frac{\partial^{2}}{\partial u^{2}}+2 \frac{\partial}{\partial u}
$$

where we introduced an exponential parametrization of $r$

$$
r=\exp u
$$

Here, $u$ varies over the whole real axis. It follows that the three dimensional diffusion of massive particles becomes one dimensional in the limit $m \rightarrow 0$. 
In the coordinates (18) it is sufficient if we restrict ourselves to the drags

$$
X=\gamma^{2} R \frac{\partial}{\partial u}
$$

The diffusion (7) is generated by

$$
\mathcal{G}=\frac{\gamma^{2}}{2}\left(\partial_{u}^{2}+2 \partial_{u}\right)+\gamma^{2} R \partial_{u} .
$$

We may write for integrals of spherically symmetric functions

$$
d \mathbf{p}=4 \pi d u \exp (3 u) .
$$

Then

$$
\mathcal{G}^{*}=e^{-3 u} \mathcal{G}^{+} e^{3 u},
$$

where

$$
\mathcal{G}^{+}=\frac{\gamma^{2}}{2}\left(\partial_{u}^{2}-2 \partial_{u}\right)-\gamma^{2} \partial_{u} R
$$

is the adjoint of $\mathcal{G}$ in $L^{2}(d u)$. The probability distribution evolves according to eq.(9). The invariant measure $d x d \mathbf{p} \Phi_{R} \equiv d x d u \Phi_{I}$ solves an analog of eq.(10)

$$
\mathcal{G}^{*} \Phi_{R}=\mathcal{G}^{+} \Phi_{I}=0
$$

where

$$
\Phi_{I}=\exp (3 u) \Phi_{R} .
$$

\section{Linearization of the Kompaneetz equation}

In this section we compare the probability distribution (9) resulting from the relativistic diffusion equation with the density of photons moving through an electron gas and described by the Kompaneetz equation. We assume that $\Phi_{E R}$ in eqs.(14)-(15) is the Bose-Einstein equilibrium distribution. This is also the equilibrium solution of the Kompaneetz equation. We show that if the density of photons is low (so that the quantum bunching effects can be neglected) then the relativistic diffusion equation (9) and the Kompaneetz equation linearized around the equilibrium coincide. We begin with the relativistic diffusion (9). The $x^{0}$ independent solution of eq.(24) is denoted $\Phi_{E I}$. The drift $R$ is related to $\Phi_{E I}$ (it is defined by $\Phi_{E R}$ in eq.(12))

$$
R=\frac{1}{2}\left(\partial_{u} \ln \Phi_{E I}-2\right) .
$$

If the diffusion (9) starts from an initial distribution

$$
\Phi_{I}=\Phi_{E I} \Psi^{I}
$$


then using the relation (26), we obtain the evolution of $\Psi$

$$
\partial_{\tau} \Psi_{\tau}^{I}=\frac{1}{2} \gamma^{2} \partial_{u}^{2} \Psi_{\tau}^{I}+\frac{1}{2} \gamma^{2}\left(\partial_{u} \ln \Phi_{E I}\right) \partial_{u} \Psi_{\tau}^{I}
$$

First, let us consider Jüttner equilibrium distribution [25] in $L^{2}(d u)$ (the classical limit of the Bose-Einstein distribution)

$$
\Phi_{E I}^{J}=r^{3} \exp (-\beta c r) \equiv r^{3} n_{J} .
$$

Then, from eq.(26)

$$
R=-\frac{1}{2}(-1+\beta c \exp (u))
$$

The diffusion generator corresponding to the Jüttner distribution reads

$$
\begin{aligned}
& \mathcal{G}=\frac{1}{2} \gamma^{2}\left(\partial_{u}^{2}+3 \partial_{u}-\beta c \exp (u) \partial_{u}\right) \\
& =\frac{1}{2} \gamma^{2}\left(r^{2} \partial_{r}^{2}+4 r \partial_{r}-\beta c r^{2} \partial_{r}\right) .
\end{aligned}
$$

For the Bose-Einstein distribution (in Compton scattering the photon number is preserved; if an equilibrium is achieved, then the chemical potential $\mu \neq 0$, see $[7])$

$$
\Phi_{E I}^{B}=r^{3}(\exp (\beta(\mu+c r))-1)^{-1} \equiv r^{3} n_{E}(\mu)
$$

we have

$$
R=-\frac{1}{2} \gamma^{2}\left(-1+\beta c \exp (u)(1-\exp (-\beta \mu-\beta c \exp u))^{-1}\right) .
$$

Eq.(28) for an evolution of $\Psi^{I}$ (with the drift (33)) in the $r$-coordinates reads

$$
\partial_{\tau} \Psi^{I}=\frac{1}{2} \gamma^{2} r^{-2}\left(\partial_{r} r^{4} \partial_{r} \Psi^{I}+r^{4}\left(\partial_{r} \ln n_{E}\right) \partial_{r} \Psi^{I}\right)
$$

where

$$
\partial_{r} \ln n_{E}=-\beta c(1-\exp (-\beta \mu-\beta c r))^{-1} .
$$

In the high energy limit we have in the diffusion equation (34)

$$
\partial_{r} \ln n_{E} \rightarrow-\beta c
$$

(the same as for the Jüttner distribution).

Now, we can compare the relativistic diffusion equation with the (non-linear) Kompaneetz equation usually written in the form [6]

$$
\partial_{\tau} n=\kappa^{2} \rho^{-2} \partial_{\rho}\left(\rho^{4}\left(\partial_{\rho} n+n+n^{2}\right)\right) .
$$

In order to explore the appearance of friction in eq.(36) we expand the photon distribution of the Kompaneetz equation around its equilibrium value 
in the same way as we expanded the distribution of the diffusion process (15) around the equilibrium in eq.(28). If we neglect $n^{2}$ on the rhs of eq.(36) then the rhs disappears (because $\partial_{\rho} n_{J}=-n_{J}$, with $\rho=\beta c r$ ) for the Jüttner distribution. Let

$$
n=\exp (-\rho) \chi
$$

Then, neglecting the $n^{2}$ term in the Kompaneetz equation (36) we obtain (after an elementary rescaling $r \rightarrow \beta c r=x, \kappa^{2}=\frac{1}{2} \gamma^{2}$ ) the diffusion equation (34) with $n_{E} \rightarrow n_{J}$.

In general, the Kompaneetz distribution $n$ equilibrates to $n_{E}$ (32) (as $\partial_{\rho} n_{E}+$ $n_{E}+n_{E}^{2}=0$ on the rhs of eq.(36)). In more detail, let us write the initial condition for the Kompaneetz equation in the form

$$
n=n_{E} \chi
$$

then the evolution of $\chi$ is determined by the equation

$$
\partial_{\tau} \chi_{\tau}=\kappa^{2} \rho^{-2}\left(\partial_{\rho} \rho^{4} \partial_{\rho} \chi_{\tau}+\rho^{4}\left(\partial_{\rho} \ln n_{E}\right) \partial_{\rho} \chi_{\tau}\right)+\kappa^{2} \rho^{-2} n_{E}^{-1} \partial_{\rho}\left(\rho^{4} n_{E}^{2}\left(\chi_{\tau}^{2}-\chi_{\tau}\right)\right)
$$

The last term in eq.(37) is absent in eq.(34). It describes the bunching effect of the quantum statistics which promotes bosons to condense. As long as the density of photons is low this term is negligible.

The Kompaneetz equation finds applications in astrophysics [1][26][27] [28][29][30] [31][32][33]. In particular, the CMBR photons described by $n_{E}(\mu=0)$ are scattered when passing clusters of galaxies and other reservoir of hot plasma. When applied to the Sunyaev-Zeldovitch effect the Kompaneetz equation is usually discussed only in a linear approximation. In such a case

$$
\Psi_{\tau}(r)=\int d y y^{-1} P_{\tau}(r, y) \Psi(y)=\left(2 \pi \gamma^{2} \tau\right)^{-\frac{1}{2}} \int_{0}^{\infty} \frac{d y}{y} \Psi(y) \exp \left(-\frac{1}{2 \tau \gamma^{2}}\left(\ln \frac{y}{r}-\frac{3}{2} \gamma^{2} \tau\right)^{2}\right)
$$

is the solution of the equation

$$
\partial_{\tau} \Psi_{\tau}=\frac{1}{2} \gamma^{2} r^{-2} \partial_{r} r^{4} \partial_{r} \Psi_{\tau} \equiv \mathcal{G}^{*} \Psi_{\tau}
$$

with the initial condition $\Psi$. The solution (38) is discussed in [27][32]. For small $\tau$ and the initial condition $\Psi=n_{E}(\mu=0)$ the solution can be approximated by

$$
\Psi_{\tau}=n_{E}(\mu=0)+\tau \mathcal{G}^{*} n_{E}(\mu=0) .
$$

The discussion of Sunyaev-Zeldovich CMBR spectrum distortion [27][30][31][32][34] is usually restricted to this approximation. The Kompaneetz equation has been derived from the Boltzmann equation [6][1] under an assumption that the electron velocities are non-relativistic. The diffusion limit of the Boltzmann equation with relativistic corrections to the Compton scattering have been calculated in [9][31][35]. 


\section{A relation to the imaginary time quantum me- chanics}

In this section we show that the (linear) relativistic diffusion equation can be solved by means of the methods of quantum mechanics. We can express solutions of the diffusion equations by an imaginary time evolution generated by a quantum mechanical Hamiltonian. The diffusion generator is of the form

$$
\mathcal{G}=\frac{1}{2} \gamma^{2} \partial_{u}^{2}-\omega \partial_{u}
$$

( we denote a general drift by $\omega$ here in order to distinguish it from particular $R$ of the earlier sections). Let

$$
\Omega(u)=\int^{u} \omega
$$

Then

$$
\exp (-\Omega) \mathcal{G} \exp (\Omega) \equiv-H=\frac{1}{2} \gamma^{2} \partial_{u}^{2}-V
$$

where

$$
V=\frac{1}{2} \omega^{2}-\frac{1}{2} \partial_{u} \omega
$$

Let

$$
\psi=\exp (-\Omega) \phi
$$

Then, from eq.(41) it follows

$$
\phi_{\tau}(u)=(\exp (\tau \mathcal{G}) \phi)(u)=\exp (\Omega(u))(\exp (-\tau H) \psi)(u)
$$

Inserting $\phi=1$ in eq.(44) we obtain $\exp (-\tau H) \exp (-\Omega)=\exp (-\Omega)$. Hence,

$$
H \exp (-\Omega)=0
$$

For the Jüttner model when

$$
\omega=-\frac{3}{2} \gamma^{2}+\frac{1}{2} \beta c \gamma^{2} \exp u
$$

eq.(42) gives

$$
V=\frac{9}{8} \gamma^{4}+\frac{1}{8} \gamma^{4} \beta^{2} c^{2} \exp (2 u)-\frac{1}{4}\left(1+3 \gamma^{2}\right) \gamma^{2} \beta c \exp (u) .
$$

This is a soluble model (the Morse potential ). The eigenvalue equation for $H$ has a solution in terms of hypergeometric functions [36]. The propagation kernel determining the time evolution (44) is also known. The eigenstate of $H$ with the zero eigenvalue is

$$
\exp (-\Omega)=\exp \left(\frac{3}{2} u-\frac{1}{2} \beta c \exp (u)\right) .
$$


The probability density in the state (47)

$$
\exp (-2 \Omega(u))=r^{3} \exp (-\beta c r)=\Phi_{E I}^{J}
$$

is just the Jüttner distribution.

For Bose-Einstein equilibrium distribution we have $\omega=-\gamma^{2}-\gamma^{2} R$ where $R$ is defined in eq.(33). It can be seen from eq.(45) that in general

$$
\exp (-2 \Omega)=\Phi_{E I}
$$

for any equilibrium distribution $\Phi_{E I}$.

\section{Outlook}

There is the unique generalization of the relativistic diffusion equation (3) to an arbitrary metric [17] describing a diffusion in the presence of gravity (some other generalizations are discussed in [37][38]). This is the diffusion which preserves the mass shell $g^{\mu \nu} p_{\mu} p_{\nu}=m^{2} c^{2}$ ( $g^{\mu \nu}$ denotes the metric tensor). In [22] we have shown that an addition of a friction is necessary if the particle energy is to be bounded in time (then it equilibrates). The diffusion equation (3) (without friction) is explicitly Lorentz invariant as follows from eq.(6). However, the equilibrium measure cannot be Lorentz invariant if it is to be normalizable (in a finite volume) and if the mass shell condition (1) is to be satisfied (there is only one invariant measure on the mass shell, this is $d \mathbf{p} p_{0}^{-1}$ ). The equilibration to the Jüttner or Bose-Einstein distribution takes place in a preferred Lorentz frame [39][40]. In a general frame described by the four-velocity $w^{\nu}$ we should write the equilibrium distribution in the form

$$
n_{E}(w, \mu)=\left(\exp \left(\beta w^{\nu} p_{\nu}+\beta w^{\nu} \mu_{\nu}\right)-1\right)^{-1}
$$

where (by convention) in the rest frame $w=(1,0,0,0)$ and $\mu_{0}=\mu$ ( $\mu_{k}=0$ for $k=1,2,3)$. In general, all the parameters $(\beta, \mu, w)$ can depend on the position $x$ (see [41] for such a framework in the description of the quark-gluon plasma).

There is the unique way to generalize the Kompaneetz equation for a photon distribution function $N(x, p)$ to an arbitrary frame on the pseudoriemannian manifold in such a way that in the rest frame on Minkowski space-time its linearized version coincides with the relativistic diffusion (9). The generalization of eq.(36) reads

$$
\begin{aligned}
& D_{\tau} N-g^{\alpha \nu} p_{\alpha} \partial_{\nu}^{x} N=\frac{\gamma^{2}}{2}\left(\triangle_{H} N\right. \\
& \left.+2 i w_{\nu} L^{\nu \rho} p_{\rho} N(N+1)+2 w^{\nu} p_{\nu} N(N+1)\right)
\end{aligned}
$$

where

$$
D_{\tau}=\partial_{\tau}-Y=\partial_{\tau}-\Gamma_{\alpha \sigma}^{j} p^{\alpha} p^{\sigma} \partial_{j}
$$


Here, $\Gamma$ are the Christoffel symbols and $D$ is the covariant derivative on the cotangent bundle describing the geodesic motion. $L_{\mu \nu}(x)$ are the generators of the Lorentz group at the point $x$ on the manifold and $p$ are the coordinates of the cotangent bundle (the momenta, see [22])

$$
L_{j k}=-i\left(g_{j l} p_{k} \partial^{l}-g_{k l} p_{j} \partial^{l}\right)
$$

and

$$
L_{0 j}=-i g_{j l}|\mathbf{p}| \partial^{l}
$$

The lhs of eq.(50) describes the well-known collisionless Boltzmann equation [42]. The operator $\triangle_{H}(2)$ is the same as the one in the relativistic diffusion equation (3). It does not depend on the metric (it is invariant under diffeomorphisms of the momenta). In eq.(50) we suggest that the collision term resulting from the Compton scattering (which could be calculated on the curved manifold following the elementary derivation in [1]) has a trivial metric dependence. In the rest frame on the Minkowski space-time eq.(50) coincides with eq.(36). In an arbitrary frame $N_{\tau}$ has the Bose-Einstein distribution $n_{E}(w, \mu)$ as the equilibrium measure.

In general, in astrophysics we have moving reference frames and non-zero gravitational fields. The effects of gravity seem to show no observational consequences unless the photons move in an electron gas around large compact massive objects. We have been concerned with the evolution at a large time in this paper. In astrophysical applications the behavior of the diffusion at large time (apart from its final effect: the equilibration) has not been discussed. However, with the increasing sophistication of the astrophysical observations the gravitational attraction as well as the large time effects of Compton scattering may give important information about the sources of the the CMBR distortions and the spectrum of light coming from compact stars.

\section{References}

[1] G.B. Rybicki and A.P. Lightman, Radiative Processes in Astrophysics,Wiley-VCH,1979

[2] S. Dodelson, Modern Cosmology, Academic Press, New York,2003

[3] E.M. Lifshits and L.P. Pitaevskii, Physical Kinetics, Pergamon Press, Oxford, 1981

[4] B. Svetitsky, Phys.Rev.D37,2484(1988)

[5] H. Van Hess, V. Greco and R.Rapp, Phys.Rev.C73,034913(2006)

[6] A.S. Kompaneetz, JETP,47,1939(1956) (in Russian) 
[7] R. Weymann,Phys.Fluids 8,2112(1965)

[8] T.A. Ensslin and C.R. Kaiser, Astron.Astrophys.360,417(2000)

[9] N. Itoh, Y. Kohyama and S. Nozawa, Astrophys.J.502,7(1998)

[10] G. Schay, PhD thesis, Princeton University, 1961

[11] R. Dudley, Arkiv for Matematik,6,241(1965)

[12] R. Hakim, Journ.Math.Phys.9,1805(1968);

[13] F. Debbasch and J.P. Rivet, Journ.Stat.Phys.90,1179(1998)

[14] J. Dunkel and P. Hänggi, Phys.Rev.E72,036106(2005)

[15] J. Dunkel, P. Talkner and P. Hänggi, Phys.Rev.D75,043001(2007)

[16] O. Oron and L.P. Horwitz, Found.Phys.35,1181(2005)

[17] J. Franchi and Y. Le Jan, Comm.Pure Appl.Math.60,187(2007)

[18] J. Dunkel and P. Hänggi,Phys.Rep.471,1(2009)

[19] C. Chevalier and F. Debbasch, Journ.Math.Phys.49,043303(2008)

[20] Z. Haba, Phys.Rev.E79,021128(2009)

[21] Z. Haba, Journ.Phys.A42,445401(2009)

[22] Z.Haba, arXiv:0909.2880

[23] L. Philpott, F. Dowker and R.D. Sorkin, arXiv:0810.5591

[24] G. Chacon-Acosta and G.M. Kremer, Phys.Rev.E76,021201(2007)

[25] F. Jüttner, Ann.Phys.(Leipzig)34,856(1911)

[26] P.J.E. Peebles, Physical Cosmology, Princeton University Press,1971

[27] R.A. Sunyaev and Ya.B. Zeldovich, Astroph.Space.Sci.7,20(1970)

[28] R. Sunyaev and L.G. Titarchuk, Astron.Astrophys. 86,121(1980) 
[29] Y.E. Lyubarsky and R.A. Sunyaev, Astron.Astrophys.123,171(1983)

[30] M.Birkshaw,Phys.Rep.310,97(1999)

[31] A. Stebbins, arXiv:astro-ph/9705178

[32] J. Bernstein and S. Dodelson, Phys.Rev.D41,354(1990)

[33] W. Hu, D. Scott and J. Silk, Phys.Rev.D49,648(1994)

[34] J.E.Carlstrom, G.P. Holder and E.D. Reese, Ann.Rev.Astr.Astrophys.40,643(2002)

[35] A. Challinor and A. Lasenby, Astroph.J.499,1(1998)

[36] S. Flügge, Practical Quantum Mechanics,Springer, Berlin,1974

[37] F. Debbasch, Journ.Math.Phys.45,2744(2004)

[38] C. Chevalier and F. Debbasch, Journ.Math.Phys.48,023304(2007)

[39] J.H. Eberly and A.Kujawski, Phys.Rev.155,10(1967)

[40] H.A. Weldon, Phys.Rev.D26,1394(1982)

[41] T. Matsui,B. Svetitsky and L.D. McLerran, Phys.Rev.D34,783(1986)

[42] J.M. Stewart, Non-equilibrium Relativistic Kinetic Theory,Lect.Notes in Physics,Vol.10,Springer,1971 